\title{
O papel da amamentação ineficaz na gênese da obesidade infantil: um aspecto para a investigação de enfermagem
}

\author{
Lack or inadequate breastfeeding as a contributing factor to child obesity: a potential problem that \\ warrents further nursing investigation \\ El papel de la lactancia materna ineficaz, en la génesis de la obesidad infantil: un aspecto para la \\ investigación de enfermería
}

\section{Márcio Flávio Moura de Araújo', Eveline Pinheiro Beserra1, Emilia Soares Chaves ${ }^{3}$}

\section{RESUMO}

Objetivo: Verificar a amamentação ineficaz como um fator que possibilitaria a gênese da obesidade infantil. Métodos: Foram avaliadas 90 crianças entre dois e cinco anos de idade numa creche de Fortaleza. Resultados: Essa avaliação revelou a seguinte condição nutricional: $57,7 \%$ (eutróficas), 14,4\% (com sobrepeso), 13,3\% (obesas), 11,1\% (com baixo peso) e 3,3\% (desnutridas). O cenário que envolvia as crianças com sobrepeso ou obesidade foi o seguinte: $60 \%$ tiveram um padrão de amamentação ineficaz (< 6 meses e não mamou); $60 \%$ viviam em famílias com uma renda mensal de menos de um salário mínimo. Conclusão: $O$ estudo possibilitou visualizar o aleitamento materno como uma ação importante no cenário da prevenção da obesidade infantil.

Descritores: Leite materno; Aleitamento materno; Obesidade; Risco; Criança

\begin{abstract}
Objective: To examine whether lack or inadequate breastfeeding is an important contributing factor to child obesity. Methods: A convenience sample of 90 children, aged two to five from a day care center in Fortaleza, Brazil, participated in this study. Results: The study's finding revealed the following: The majority of the children $(57.7 \%)$ were well nourished. The remainder of the children were overweight (14.4\%), obese (13.3\%, underweight (11.1\%), and undernourished (3.3\%). A further evaluation of the data of overweight and obese children revealed that: $60 \%$ had not been breastfed or were breastfed for a period inferior to six months; and, their family had minimum wage income. Conclusion: The study suggests that breastfeeding is a very important factor to consider when addressing child obesity preventive interventions.
\end{abstract}

Keywords: Milk, human; Breast feeding; Obesity; Risk; Child

\section{RESUMEN}

Objetivo: Verificar el amamantamiento ineficaz como factor que posibilitaría la génesis de la obesidad infantil. Métodos: Fueron evaluados 90 niños entre dos y cinco años de edad en una guardería de Fortaleza. Resultados: Esa evaluación reveló la siguiente condición nutricional: el 57,7\% eutróficas, el 14,4\% con sobrepeso, el 13,3\% obesas, el 11,1\% con bajo peso y el 3,3\% desnutridas. El escenario que involucraba a los niños con sobrepeso u obesidad fue el siguiente: el 60\% tuvo un patrón de amamantamiento ineficaz ( $<6$ meses y no mamó); el 60\% vivía en familias con un ingreso mensual de menos de un sueldo mínimo. Conclusión: Así, la lactancia materna es una acción importante en el escenario de la prevención de la obesidad infantil.

Descriptores: Leche humana; Lactancia materna; Obesidad; Riesgo; Niño

1 Acadêmico de Enfermagem do $6^{\circ}$ semestre da Universidade Federal do Ceará - UFC - Fortaleza (CE), Brasil.

${ }^{2}$ Doutora do Curso de Pós-Graduação em Enfermagem da Universidade Federal do Ceará - UFC - Fortaleza (CE), Brasil.. 


\section{INTRODUÇÃO}

A estabilização na disponibilidade dos alimentos, iniciada no período histórico da antiguidade, com o domínio das técnicas agrícolas, foi um marco na vitória do homem contra a morbi-mortalidade desencadeada pela fome, característica daquela época, e, conseqüentemente, um passo decisivo para a ascensão e manutenção da espécie humana. Todavia, mesmo nesse período no qual a inanição era predominante, já se encontravam, esporadicamente, casos de sobrepeso e obesidade na população. Atualmente, na era da globalização, houve uma inversão desse quadro: a fome diminuiu, mas continua causando mortes em países subdesenvolvidos, enquanto a obesidade é considerada uma pandemia mundial e importante problema de saúde pública em países desenvolvidos e subdesenvolvidos.

No Brasil, recentes estudos realizados pelo Instituto Brasileiro de Geografia e Estatística (IBGE), juntamente com o Ministério da Saúde, sobre a composição da dieta alimentar e do estado nutricional, com 95,5 milhões de brasileiros acima de vinte anos, de todo o país, revelaram: 40,6\% (cerca de 38,8 milhões) de pessoas apresentam sobrepeso, sendo que destas, $11 \%$ (cerca de 10,5 milhões) apresentaram obesidade. Isso contra $4 \%$ (cerca de 3,8 milhões) de pessoas, que apresentaram déficit de peso ${ }^{(1)}$. Logo, a freqüência de excesso de peso na população brasileira adulta supera em dez vezes àquela que apresenta déficit de peso.

Na população infantil, estudos estimam que, no Brasil, haja cerca de três milhões de crianças, com idade inferior a 10 anos, apresentando excesso de peso. Destes casos, $95 \%$ estariam relacionados à má alimentação, enquanto, apenas $5 \%$ seriam decorrentes de fatores endógenos. Outro aspecto importante é o fato de que, apesar desta doença ser ainda prevalente em crianças da classe média e alta, é crescente o sua ascensão em crianças pobres ${ }^{(2)}$.

A obesidade infantil é uma enfermidade crônica que se perpetua na vida desta população, na maioria das vezes até a fase adulta. Estima-se que cerca de $80 \%$ das crianças obesas serão também obesas quando adultas. Estas serão, em sua grande maioria, acometidas por transtornos metabólicos que desencadearão no futuro problemas metabólicos como hipertensão arterial, diabetes, dislipidemias e doenças cardiovasculares, principalmente as isquêmicas (infarto, trombose, embolia, aterosclerose, etc. $)^{(3)}$. A obesidade infantil pode ainda favorecer $\mathrm{o}$ surgimento de problemas ortopédicos, apnéia do sono, alguns tipos de cânceres e distúrbios psicológicos. Todos esses problemas causam má qualidade de vida, oneração aos cofres públicos, por meio de tratamentos, internações ou até mesmo levar o indivíduo à morte.

A adiposidade na infância, em sua grande parte, não está diretamente relacionada ao consumo excessivo de alimentos, mas sim ao consumo de alimentos hipercalóricos, atrelado a hábitos de vida sedentários. A construção deste padrão alimentar e comportamental tem como intermediador fatores gestacionais, familiares e sócio-culturais.

Estudos científicos têm sugerido que as primeiras experiências nutricionais de um indivíduo não guardam relações somente com o seu peso quando adulto, mas também podem interferir na sua suscetibilidade a determinadas doenças crônicas na fase adulta como a obesidade, hipertensão arterial, doenças cardiovasculares e o diabetes tipo 2. Tal fenômeno vem sendo denominado "imprinting metabólico". Este é compreendido como um fenômeno pelo qual uma experiência nutricional precoce, agindo durante um período crítico e específico do desenvolvimento humano, pode acarretar um efeito duradouro por toda a vida do indivíduo, interferindo na sua suscetibilidade a determinadas doenças ${ }^{(4)}$.

$\mathrm{Na}$ dinâmica fisiológica e emocional da criança, a amamentação é uma das primeiras experiências nutricionais do recém-nascido, que antes recebia uma nutrição intra-uterina. O leite materno é composto por inúmeros fatores bioativos como hormônios e enzimas que vão atuar sobre o crescimento, diferenciação e maturação funcional de órgãos específicos, afetando, diretamente, o desenvolvimento do infante. A composição ímpar do leite materno poderia, portanto, estar implicada em um processo de "imprinting metabólico", alterando, por exemplo, o número e/ou tamanho dos adipócitos ou estimulando o processo de homeostase metabólica. Os adipócitos estão envolvidos na regulação do balanço energético que, quando alterado, pode possibilitar um cenário para a instalação da obesidade na infância ${ }^{(5)}$.

Dessa forma, sabendo que o enfermeiro é um dos principais profissionais envolvidos na questão do aleitamento materno e saúde do escolar, este estudo objetivou investigar o papel da amamentação ineficaz na gênese da obesidade infantil em um grupo de crianças provenientes de uma creche da cidade de Fortaleza.

\section{MÉTODOS}

Trata-se de um estudo do tipo transversal com uma abordagem quantitativa-descritiva. Nesse modelo há uma investigação sobre as dimensões e manifestações de um determinado fenômeno numa população específica ${ }^{(6)}$.

O local destinado para a realização da pesquisa foi um centro educacional infantil da cidade de Fortaleza, vinculado à Secretaria de Ação Social da prefeitura do município. $\mathrm{O}$ estudo foi desenvolvido durante o período de setembro a novembro de 2005. A amostra foi constituída pelas 90 crianças, na faixa etária de dois a cinco anos, matriculadas nesta instituição. 
O instrumento utilizado para a coleta de dados foi um formulário, contendo questões referentes a dados pessoais e antropométricos (peso e altura) da criança. $\mathrm{O}$ padrão antropométrico de referência adotado foi o do National Center for Health Statistic (NCHS), com análise segundo o critério de percentil, conforme as recomendações da Organização Mundial de Saúde (OMS $)^{(7)}$. O mesmo foi aplicado somente após a assinatura do Termo de Consentimento Livre e Esclarecido pelas mães das crianças pesquisadas.

O estado nutricional, por meio do critério de percentil, foi assim diagnosticado: eutrofia (percentil no intervalo entre 10-85); sobrepeso (percentil no intervalo $>85$ e $<95$ ); obesidade (percentil e $>95$; baixo peso percentil compreendido entre $<10$ e $>5$ ); a desnutrição foi diagnosticada com percentil $<5$.

A pesagem das crianças foi realizada numa balança aferida com capacidade de $120 \mathrm{~kg}$, com as mesmas vestindo somente calção e em posição ereta. Para a mensuração da estatura, tomaram-se alguns cuidados no momento da coleta, a saber: as crianças foram colocadas descalças, em posição ereta, encostadas numa superfície plana vertical (local de fixação da fita métrica), braços pendentes com as mãos espalmadas sobre as coxas, os calcanhares foram unidos e as pontas dos pés afastadas formando um ângulo de $60^{\circ}$, joelhos postos em contato, cabeça ajustada ao plano de Frankfurt e em inspiração profunda.

Posteriormente, construiu-se um banco de dados no programa Microsoft Excel 2000 para a tabulação, e conseqüente análise das informações antropométricas. Com relação às crianças em que se detectou sobrepeso ou obesidade, houve a convocação das respectivas mães para uma reunião individual, a fim de se captar, destas, as informações acerca do período de amamentação e situação socioeconômica das crianças com excesso de peso. Os demais resultados encontrados foram analisados mediante literatura pertinente com a temática.

O presente estudo foi desenvolvido dentro dos padrões éticos da pesquisa envolvendo seres humanos, preconizados pela Resolução n. ${ }^{\circ}$ 196/96 do Conselho Nacional de Saúde, tendo sido aprovado pelo Comitê de Ética em Pesquisa, do Complexo Hospitalar da Universidade Federal do Ceará, sob o protocolo de $\mathrm{n}^{\mathrm{0}} 258 / 05$.

\section{RESULTADOS}

Dentre as 90 crianças avaliadas, $58(60,4 \%)$ eram do sexo masculino e $32(35,6 \%)$ do sexo feminino. A avaliação nutricional dessas crianças, segundo o critério de percentil, evidenciou os seguintes parâmetros: $57,7 \%$ (52/90) encontravam-se eutróficas; $11,1 \%$ (10/90) com baixo peso; $3,3 \%$ (3/90) desnutridas, enquanto $14,4 \%$
$(13 / 90)$ e $13,3 \%(12 / 90)$ apresentaram, respectivamente, sobrepeso e obesidade (Tabela 1).

Tabela 1 - Distribuição das crianças, segundo estado nutricional. Fortaleza-CE. 2005

\begin{tabular}{llrr}
\hline Estado Nutricional & Percentil & N & $\mathbf{0 \%}$ \\
\hline Eutrofia & $10-85$ & 52 & 57,7 \\
Sobrepeso & $>85<90$ & 13 & 14,4 \\
Obesidade & $>90$ & 12 & 13,3 \\
Baixo peso & $<10>5$ & 10 & 11,1 \\
Desnutrição & $<5$ & 3 & 3,3 \\
\hline Total & & $\mathbf{9 0}$ & $\mathbf{1 0 0 , 0}$ \\
\hline
\end{tabular}

A prevalência de sobrepeso e obesidade foi maior nos meninos, ocorrendo numa percentagem de 15,5\% para ambas as situações nutricionais de acúmulo de peso, enquanto que nas meninas essas taxas foram $12,5 \% \mathrm{e}$ 9,3\%, respectivamente, para sobrepeso e obesidade.

O período de aleitamento materno nas crianças com diagnóstico de sobrepeso ou obesidade foi assim caracterizado: 48\% (12/25) mamaram menos de seis meses; $36 \%(9 / 25)$ mamaram seis meses ou mais; $12 \%$ (3/25) não mamaram e 4\% (1/25) ainda mamavam. Sendo assim, $60 \%$ das crianças com excesso de peso (sobrepeso ou obesidade) do estudo apresentaram um padrão de amamentação ineficaz, segundo critérios da OMS que estabelecem um período de amamentação de seis meses (Tabela 2).

Tabela 2 - Período de amamentação das crianças com sobrepeso ou obesidade. Fortaleza-CE. 2005

\begin{tabular}{lcrr}
\hline $\begin{array}{l}\text { Período de } \\
\text { amamentação }\end{array}$ & $\begin{array}{c}\text { Avaliação da } \\
\text { amamentação }\end{array}$ & N & $\mathbf{\%}$ \\
\hline$<6$ meses & Ineficaz & 12 & 48,0 \\
$>6$ meses & Eficaz & 9 & 36,0 \\
Não mamou & Ausente & 3 & 12,0 \\
Ainda mama & - & 1 & 4,0 \\
\hline Total & & $\mathbf{2 5}$ & $\mathbf{1 0 0 , 0}$ \\
\hline
\end{tabular}

Os resultados acerca da condição econômica das famílias das crianças com acúmulo de peso revelaram: $60 \%(15 / 25)$ viviam, mensalmente, com menos de um salário mínimo; $28 \%(7 / 25)$ viviam com 1 a 3 salários e $12 \%(3 / 25)$ com 3 ou mais salários mensais.

\section{DISCUSSÃO}

Apesar desta pesquisa não ter controlado outras variáveis que poderiam interferir no estado nutricional infantil como: peso da criança ao nascer, ingestão calórica atual desta, nível de atividade física, entre outras, os resultados desta investigação nos sugerem que uma 
amamentação ineficaz, atrelada a uma condição socioeconômica deficiente, pode favorecer o surgimento de um cenário propício para a gênese da obesidade infantil. O papel do aleitamento materno na prevenção do excesso de peso pediátrico é uma hipótese difundida mundialmente e respaldada em vários trabalhos científicos.

Em um estudo seccional realizado na Alemanha com uma amostra de 9.357 crianças na faixa etária entre cinco e seis anos de idade, publicado em 2000, foi detectado uma prevalência de obesidade de $4,5 \%$ entre as crianças que nunca haviam sido amamentadas e de $2,8 \%$ naquelas que receberam aleitamento materno. Observou-se, também, neste estudo que quanto maior foi o período de amamentação, menor foi a prevalência de obesidade: $3,8 \%$ para as que mamaram por dois meses, $2,3 \%$ nas que mamaram por três a cinco meses, $1,7 \%$ em crianças que mamaram seis a doze meses e $0,8 \%$ nas crianças que foram aleitadas por mais de 12 meses ${ }^{(8)}$. Outro estudo alemão com 2.108 crianças entre 9 e 10 anos de idade chegou a mesma conclusão: a prevalência de adiposidade infantil é menor nas crianças com uma amamentação eficaz (seis meses ou mais) ${ }^{(9)}$.

Outros dois estudos epidemiológicos europeus de base populacional acentuam o caráter imune do leite materno contra o excesso de peso pediátrico. $\mathrm{O}$ primeiro que avaliou 8.186 meninas e 7.155 meninos na faixa etária de 9 a 14, detectou que o risco para sobrepeso foi menor nos pesquisados que receberam aleitamento materno até o sexto mês do que naqueles em que foram adotadas fórmulas lácteas artificiais. $\mathrm{O}$ segundo estudo, realizado na República Theca com 33.768 crianças entre 6 a 14 anos de idade, constatou uma prevalência de sobrepeso e obesidade menor entre aqueles que receberam aleitamento materno exclusivo até o sexto mês ${ }^{(10-11)}$.

Da mesma forma que nesses trabalhos supracitados, verificou-se, no cenário deste estudo, que a amamentação ineficaz foi um comportamento que, atrelado a outras condições exógenas, possibilitou a gênese do excesso de peso na população infantil, já que, em $60 \%$ dos pesquisados com um quadro de sobrepeso ou obesidade, verificou-se haver um padrão de amamentação ineficaz (menor que seis meses), ou ausente.

Os mecanismos pelos quais o leite materno desempenharia uma proteção em relação à obesidade ainda não foram totalmente elucidados. É provável que o leite materno esteja envolvido no fenômeno do "imprinting metabólico", promovendo, a partir de uma exposição do indivíduo por determinado período do individuo aos componentes do leite materno, uma diminuição na suscetibilidade deste torna-se obeso na infância e na adultícia. Todavia, sabe-se também que o néctar da genitora é composto por fatores bioativos como os hormônios insulina, $\mathrm{T}_{3}$ e $\mathrm{T}_{4}$ e a leptina, que agem no centro da alimentação e saciedade, localizado no hipotálamo, regulando o balanço energético do metabolismo infantil ${ }^{(4-5)}$.

Especialmente a leptina, ingerida pela criança durante a amamentação, é responsável pelo início da regulação e homeostase energética infantil. Esse hormônio peptídico atua no metabolismo do lactente, inibindo, no hipotálamo, o apetite e as vias anabólicas, e estimulando as catabólicas ${ }^{(4,12-14)}$. Por conseguinte, a leptina pode constituir um meio importante pelo qual se sinaliza ao cérebro que houve armazenamento suficiente de energia, diminuindo assim o apetite e a necessidade de ingestão de alimentos.

Outro aspecto importante, e uma das possíveis causas das taxas, nas crianças, de $14,4 \%$ e $13,3 \%$ de sobrepeso e obesidade, respectivamente, é que essa amamentação ineficaz esteve, provavelmente, combinada à adoção de fórmulas lácteas para complementar ou substituir a anterior alimentação infantil.

A interrupção precoce da amamentação e a adoção da alimentação látea artificial, eleva o consumo energético infantil em $15 \%$ a $20 \%$ quando comparado ao consumo energético de crianças em aleitamento materno exclusivo. O consumo energético das crianças em amamentação é inferior aos das que adotam uma dieta artificial, todavia, o consumo quantitativo nas que mamam é superior. Isto aponta que a criança alimentada com fórmulas artificiais, mesmo com uma ingesta menor, está ingerindo uma alimentação hipercalórica em relação as que ingerem o alimento materno natural ${ }^{(15)}$. Essa exposição infantil à uma alimentação hipercalórica, na lactância, e nos primeiros anos da infância, é uma possível causa de obesidade precoce por aumento do tecido adiposo.

A constituição do tecido adiposo ocorre a partir da geração de células adiposas (adipócitos). Estas são geradas por meio de uma nutrição excessiva de carboidratos e proteínas que, quando não metabolizados, são armazenados sob a forma de gordura no tecido subcutâneo. Logo, quanto maior for o armazenamento de gordura, maior será o número de células adiposas geradas, e, conseqüentemente, de tecido adiposo. $\mathrm{O}$ fenômeno da adipogênese tem o seu início ainda na fase intra-uterina e término aos sete anos de idade. Após esse período, pode ocorrer somente uma atrofia ou hipertrofia das células adiposas, quando, respectivamente, há perda ou ganho de peso. Acredita-se que o indivíduo obeso, com um tecido adiposo excessivo, possua um ponto de ajuste mais elevado para o armazenamento de nutrientes e alcance da saciedade alimentar do que indivíduos com peso normal, promovendo, assim, um risco maior para uma hiperfagia e ganho de peso ${ }^{(14,16)}$.

Todavia, a ingestão de gordura em concentrações adequadas é algo necessário nas dietas infantis, para que 
haja uma maturação adequada do sistema nervoso e prevenção de sobrepeso. Pois, contraditoriamente, é relatado, no meio científico, que crianças com antecedentes de desnutrição apresentam deficiência na oxidação ou das gorduras (lipólise), dessa forma facilitando o seu acúmulo corpóreo. Autores argumentam, inclusive, que esse fato pode ser um dos mecanismos capazes de explicar a ascensão da obesidade em países em desenvolvimento. Porém, outros estudos sugerem que o desmame precoce seria a principal causa do aumento da prevalência do sobrepeso e da obesidade infantil em populações pobres $^{(4,15-16)}$. Desta forma, os 3,3\% dos pesquisados, com quadro de desnutrição, encontram-se também propensos a desenvolver obesidade.

A vulnerabilidade infantil frente à obesidade promovida por um padrão de amamentação ineficaz é uma temática relevante para a pesquisa e atuação terapêutica de enfermagem na prevenção dessa doença metabólica. O principal cuidado de enfermagem, frente a esta situação de aleitamento ausente ou deficiente, é a educação em saúde.

A educação em saúde é um processo que compreende a transmissão de conhecimentos relativos à conquista da saúde visando a mudança de comportamento e estilo de vida, que anteriormente eram de vulnerabilidade ou nocivo à saúde para um promotor da saúde. Nesse processo de mudanças, o indivíduo passa a ser o principal responsável pelo seu estado de saúde

As nutrizes, em sua grande maioria, são fisicamente capazes de aleitar até o sexto mês de vida infantil, desde que recebam incentivos corretos e suficientes, como também sejam protegidas de comentários desalentadores acerca do ato de aleitar, já que esse momento feminino é marcado, algumas vezes, por receio, insegurança e dor.

Nesse propósito, o enfermeiro, por intermédio da educação em saúde, assume grande relevância, pois pode, já no pré-natal, discutir com a gestante as vantagens de uma amamentação plena. No puerpério, o enfermeiro é o profissional mais engajado em educar a puérpera acerca da alimentação mais adequada durante o aleitamento, dos exercícios que facilitam a ejeção de leite e da adoção de fatores ambientais favoráveis à implementação de uma experiência prazerosa e saudável durante a amamentação $^{(17)}$.

O enfermeiro, ao realizar um cuidado educativo junto às mães que aleitam, além de reduzir uma futura vulnerabilidade em relação à obesidade infantil e adulta, quando incentiva a prática do aleitamento materno no primeiro semestre, estará trazendo apoio e tranqüilidade à mulher nesse momento ímpar que é a maternidade. Dessa forma, percebe-se que a inserção da educação em saúde ao arsenal de recursos terapêuticos disponibilizados pela enfermagem ao binômio mãe-filho, é uma ação fundamental na promoção da saúde materno-infantil brasileira, frente à ascensão cada vez maior da obesidade entre crianças.

\section{CONSIDERAÇÕES FINAIS}

Embora os resultados aqui encontrados sejam oriundos de uma investigação transversal, na qual não houve um controle de outras variáveis que poderiam contribuir para o acúmulo de peso infantil, como também por ter ocorrido numa população específica de uma creche, acredita-se, a partir dos resultados e da literatura científica encontrada, haver uma associação importante entre amamentação ineficaz e excesso de peso infantil. Dessa forma, o aleitamento materno exclusivo até o sexto mês deve ser um comportamento incentivado por meio de políticas públicas de saúde, a fim de que se possa atenuar a problemática da obesidade na infância e, conseqüentemente, na idade adulta. Tal ação repercutiria diretamente nos índices de morbi-mortalidade das várias doenças desencadeadas pela obesidade adulta, que, anualmente, oneram os cofres públicos com gastos em tratamentos e óbitos.

\section{REFERÊNCIAS}

1. Instituto Brasileiro de Geografia e Estatística. Pesquisa de orçamentos familiares-POF 2002-2003 [texto na Internet].Brasília (DF):IBGE; 2003. [citado 2005 Set 27]. Disponível em: http://www.ibge.gov.br/home/estatisica/ populacao/condicao/pof/imc

2. Corso ACT, Botelho LJ, Zeni LAZR, Moreira EAM. Sobrepeso em crianças menores de 6 anos de idade em Florianópolis, SC. Rev Nutr. 2003; 16(1): 21-8.

3. Pellanda LC, Echenique L, Barcellos LMA, Maccari J, Borges FK, Zen BL. Doença cardíaca isquêmica: a prevenção inicia durante a infância. J Pediatr (Rio J). 2002; 78(2): 91-6.

4. Balaban G, Silva GAP. Efeito protetor do aleitamento materno contra a obesidade infantil. J Pediatr (Rio J). 2004; 80(1): 7-16.

5. Balaban G, Silva GAP, Dias MLCM, Dias MCM, Fortaleza GTM, Morotó FMM, Rocha ECV. O aleitamento materno previne o sobrepeso na infância? Rev Bras Saúde Matern Infant. 2004; 4(3):263-8.

6. Polit DF, Hungler BP. Delineamento de pesquisa. In: Polit DF, Hungler BP. Fundamentos de pesquisa em enfermagem. 3a ed. Porto Alegre: Artes Médicas; 1995. p.108-40.

7. World Health Organization. Physical status: the use and interpretation of anthropometry. Geneva; WHO; 1995. 434p.

8. Von Kries R, Koletzko B, Sauerwald T, von Mutius E. Does breast-feeding protect against childhood obesity? Adv Exp Med Biol. 2000; 478:29-39.

9. Liese AD, Hirsh T, von Mutius E, Keil U, Leupold W, Weiland SK. Inverse association of overweight and breast feeding in 9 to 10 -y-old children in Germany. Int J Obes Relat Metab Disord. 2001; 25(11): 1644-50.

10. Gillman MW, Rifas-Shiman SL, Camargo CA Jr, Berkey CS, Frazier L, Rockett HR, etal. Risk of overweight among adolescents who were breastfed as infants. JAMA. 2001; 285(19):2461-7. 
11. Toschke AM, Vignerova J, Lhotska L, Osancova K, Koletzko B, von Kries R. Overweight and obesity in 6-to 14-year-old Czech children in 1991: protective effect of breast-feeding. J Pediatr. 2002; 141(6): 764-9.

12. Houseknecht KL, McGuire MK, Portocarrero CP, McGuire MA, Beerman K. Leptin is present in human milk and is related to maternal plasma leptin concentration and adiposity. Biochem Biophys Res Commun. 1997; 240(3):742-7.

13. Uysal FK, Onal EE, Aral YZ, Adam B, Dilmen U, Ardicolu Y. Breast milk leptin: its relationship to maternal and infant adiposity. Clin Nutr. 2002; 21(2): 157-60.

14. Guyton AC, Hall JE. Balanços dietéticos; regulação da alimentação; obesidade e inanição; vitaminas e sais minerais.
In: Guyton AC, Hall JE. Tratado de fisiologia médica. 10a ed. Rio de Janeiro: Guanabara Koogan; 2002. p.750-60.

15. Nejar FF, Segall-Corrêa AM, Rea MF, Vianna RPT, Panigassi G. Padrões de aleitamento materno e adequação energética. Cad Saúde Pública. 2004; 20(1): 64-71.

16. Hardy SC, Kleinman RE. Fat and cholesterol in the diet of infants and young children: implications for growth, development, and long-term health. J Pediatr. 1994; 125(5 Pt 2): S69-77. Review.

17. Wong DL. Problemas de saúde do lactente. In: Whaley LF, Wong DL. Enfermagem pediátrica: elementos essenciais à intervenção efetiva. 5a ed. Rio de Janeiro: Guanabara Koogan; 1999. p.307-10. 